\title{
THE SDE SOLVED BY LOCAL TIMES OF A BROWNIAN EXCURSION OR BRIDGE DERIVED FROM THE HEIGHT PROFILE OF A RANDOM TREE OR FOREST ${ }^{1}$
}

\author{
By Jim PitMan \\ University of California, Berkeley
}

Let $B$ be a standard one-dimensional Brownian motion started at 0 . Let $L_{t, v}(|B|)$ be the occupation density of $|B|$ at level $v$ up to time $t$. The distribution of the process of local times $\left(L_{t, v}(|B|), v \geq 0\right)$ conditionally given $B_{t}=0$ and $L_{t, 0}(|B|)=l$ is shown to be that of the unique strong solution $X$ of the Itô SDE,

$$
d X_{v}=\left\{4-X_{v}^{2}\left(t-\int_{0}^{v} X_{u} d u\right)^{-1}\right\} d v+2 \sqrt{X_{v}} d B_{v}
$$

on the interval $\left[0, V_{t}(X)\right)$, where $V_{t}(X):=\inf \left\{v: \int_{0}^{v} X_{u} d u=t\right\}$, and $X_{v}=0$ for all $v \geq V_{t}(X)$. This conditioned form of the Ray-Knight description of Brownian local times arises from study of the asymptotic distribution as $n \rightarrow \infty$ and $2 k / \sqrt{n} \rightarrow l$ of the height profile of a uniform rooted random forest of $k$ trees labeled by a set of $n$ elements, as obtained by conditioning a uniform random mapping of the set to itself to have $k$ cyclic points. The SDE is the continuous analog of a simple description of a Galton-Watson branching process conditioned on its total progeny. For $l=0$, corresponding to asymptotics of a uniform random tree, the SDE gives a description of the process of local times of a Brownian excursion which is equivalent to Jeulin's description of these local times as a time change of twice a Brownian excursion. Another corollary is the Biane-Yor description of the local times of a reflecting Brownian ridge as a timechanged reversal of twice a Brownian meander of the same length.

1. Introduction. This paper describes the local time process of a Brownian excursion or reflecting Brownian bridge as the solution of a stochastic differential equation (SDE). This equation is the continuous analog of a corresponding description of the height profile of a random tree or forest obtained by conditioning a discrete time Galton-Watson process on its total progeny. This result is a development of the deep connection between Brownian excursions and branching processes established over the past 45 years and summarized briefly in the next paragraph. In the case of Brownian excursion, the SDE description of the local time process is implicit in Jeulin [27], page 264, and a derivation of the SDE from branching process consideration was indicated by Kersting [30], page 11. The same SDE appears in

Received January 1998; revised June 1998.

${ }^{1}$ Supported in part by NSF Grant DMS-97-03961.

AMS 1991 subject classifications. Primary 60J65; secondary 60J55, 60J60, 60J80, 05C05.

Key words and phrases. Local time, Bessel process, Galton-Watson branching process, Brownian meander, Ray-Knight theorems, random mapping. 
descriptions by Leuridan [47], Section I.3, of the local time process of Brownian motion up to a fixed time. Here a unified description of the local time processes of Brownian excursion and reflecting Brownian bridge is given in terms of solutions of this SDE, and this description is applied to identify these processes as weak limits of suitably scaled height profiles of random trees and forests.

Harris [21] observed that excursions of the simple random walk embedded in a Brownian path could be recoded to form the random tree associated with a discrete time Galton-Watson branching process with geometric(1/2) offspring distribution. As pointed out by Kawazu and Watanabe [28], this branching structure of random walk excursions is implicit in Knight's approach by random walk approximation to the Ray-Knight description of Brownian local time processes [67, 34, 68]. The Ray-Knight theorems are thus linked to Feller's [20] diffusion approximation for a critical branching process. Le Gall $[41,42]$ connected these ideas to Williams' path decompositions of Brownian motion [78]. Neveu and Pitman [51, 52] showed how the family tree of a continuous time Yule process is embedded in a path governed by Itô's law of Brownian excursions conditioned to exceed a given height. Aldous [2, 3, 4] developed analogous results encoding a Brownian excursion of given length as a continuum random tree, in the context of a more general theory of continuum random trees as weak limits as $n \rightarrow \infty$ of combinatorially defined trees with $n$ vertices. See also [44]. There is much current interest in the use of Brownian and other local time processes as models of continuous state branching processes and the applications of such processes to Markovian superprocesses. See, for instance, $[43,45]$ and papers cited there.

Let $B$ denote a standard Brownian motion started at 0 . Let

$$
B^{\mathrm{br}, t}:=\left(B_{s}^{\mathrm{br}, t}, 0 \leq s \leq t\right)=_{d}\left(B_{s}, 0 \leq s \leq t \mid B_{t}=0\right)
$$

denote a Brownian bridge of length $t$. Here and throughout the paper, ":=" means "equal by definition" and " $={ }_{d}$ " denotes equality in distribution of random variables or processes. For a fixed time $T>0$, let $G_{T}:=\sup \{s: s \leq T$, $\left.B_{s}=0\right\}$ be the last zero of $B$ before time $T$ and $D_{T}:=\inf \left\{s: s \geq T, B_{s}=0\right\}$ be first zero of $B$ after time $T$. It is known that for each $0<t \leq T$ the law of

$$
\left(B_{s}, 0 \leq s \leq G_{T}\right) \text { given } G_{T}=t
$$

does not depend on $T$ and is that of $B^{\mathrm{br}, t}$, the Brownian bridge of length $t$. A process with the law of

$$
\left(|B|_{G_{T}+s}, 0 \leq s \leq D_{T}-G_{T}\right) \text { given } D_{T}-G_{T}=t,
$$

which also does not depend on $T$, is called a Brownian excursion of length $t$, denoted here by $B^{\mathrm{ex}, t}$. A process with the law of

$$
\left(|B|_{G_{T}+s}, 0 \leq s \leq T-G_{T}\right) \text { given } T-G_{T}=t,
$$

which again does not depend on $T$, is called a Brownian meander of length $t$. It is well known that these Brownian bridges, excursions and meanders of length $t$ can be constructed by Brownian scaling from the corresponding 
standard processes of length 1 . If the length of one of these processes is not mentioned, it is assumed to be 1 . See [68, 5] for background and further references to these processes.

For a suitable continuous function $f$ with domain containing $[0, t]$, let $L_{t, v}(f)$ denote the local time of $f$ up to time $t$ at level $v$ as defined by the occupation density formula

$$
\int_{0}^{t} g\left(f_{u}\right) d u=\int_{-\infty}^{\infty} g(v) L_{t, v}(f) d v
$$

for every nonnegative Borel measurable $g$ and continuity in $v$. Let $B^{|b r|, t}$ be a reflecting Brownian bridge (RBB) of length $t$,

$$
B^{|\mathrm{br}|, t}:=\left(B_{s}^{|\mathrm{br}|, t}, 0 \leq s \leq t\right):=\left(\left|B_{s}^{\mathrm{br}, t}\right|, 0 \leq s \leq t\right) .
$$

The abbreviation $L_{t, v}^{|\mathrm{br}|}:=L_{t, v}\left(B^{|\mathrm{br}|, t}\right)$ will be used throughout the paper for the local time up to time $t$ at level $v$ of a RBB of length $t$. Using the Ray-Knight description of Brownian local times, Williams' [78] time reversal theorem and an identity of $\sigma$-finite measures related to Brownian excursions, Biane and Yor [7] showed that the process $\left(L_{1, v}^{|\mathrm{br}|}, v \geq 0\right)$ of local times of RBB is a time change of the time reversal of twice a Brownian meander. Corollary 16 below recalls the precise statement of this result.

Section 3 reviews the appearance of the process $\left(L_{1, v}^{\mid b r}, v \geq 0\right)$ as the asymptotic distribution for large $n$ of the height profile of the random forest generated by a uniform random mapping of an $n$-element set to itself $[1,17]$. The density of a limit law derived by Proskurin [66] from the height profile of this random forest was identified with the density of $L_{1, v}^{|b r|}$ by Aldous and Pitman [1]. See also [72, 73] for a derivation of this density by a more straightforward random walk approximation. Recent work [17] develops the approach of [1] by use of a generating function analysis of the finite-dimensional distributions of the height profile of a random mapping. After passage to the limit, this yields a formula for the characteristic function of the finite-dimensional distributions of $\left(L_{1, v}^{|\mathrm{br}|}, v \geq 0\right)$ in terms of a contour integral in the complex plane with a rather complicated integrand. The purpose of this paper is to record another description of the process $\left(L_{1, v}^{|\mathrm{br}|}, v \geq 0\right)$ in terms of the process $X$ introduced in the following lemma, which is easily verified by techniques of stochastic calculus [68].

Lemma 1. Let $\beta$ be a Brownian motion. For each $l \geq 0$ and $t>0$ there exists a unique strong solution $X$ of the Itô $S D E$,

$$
X_{0}=l ; \quad d X_{v}=\delta_{v}(X) d v+2 \sqrt{X_{v}} d \beta_{v}
$$

where

$$
\delta_{v}(X):=4-X_{v}^{2}\left(t-\int_{0}^{v} X_{u} d u\right)^{-1}
$$


with the convention that the equation for $X$ is to be solved only on $\left[0, V_{t}(X)\right)$ and that $X_{v}=0$ for $v \geq V_{t}(X)$ where

$$
V_{t}(X):=\inf \left\{v: \int_{0}^{v} X_{u} d u=t\right\}
$$

Definition 2. For each $t>0$ and $l \geq 0$ let $X:=\left(X_{l, t, v}, v \geq 0\right)$ denote a process with the same distribution as the solution $X$ of the above SDE. Also, let $X_{0,0, v}:=0$ for all $v \geq 0$.

The following proposition records some basic properties of this process $X$, which follow easily from its definition by standard techniques of stochastic calculus.

Proposition 3. The process $X$ enjoys the following properties.

(i) For each $l \geq 0$ and $t>0$, the random time $V_{t}(X)$ is strictly positive and finite a.s., and the left limit of $X$ at time $V_{t}(X)$ exists and equals 0 a.s. Consequently, the process $X$ has continuous paths almost surely.

(ii) For each $l \geq 0$ and $t>0$,

$$
X_{l, t, 0}=l \quad \text { and } \int_{0}^{\infty} X_{l, t, v} d v=t \quad a . s .
$$

(iii) For each $t>0$, the collection of laws of $\left(X_{l, t, v}, v \geq 0\right)$ for $l \geq 0$ is determined by the collection of laws of $\left(X_{l, 1, v}, v \geq 0\right)$ for $l \geq 0$ via the formula

$$
\left(X_{l, t, v}, v \geq 0\right)={ }_{d}\left(\sqrt{t} X_{l / \sqrt{t}, 1, v / \sqrt{t}}, v \geq 0\right) .
$$

(iv) Let $E:=[0, \infty) \times(0, \infty) \cup\{(0,0)\}$ and for $w:=(l, t) \in E$ let $Q^{w}$ denote the law of the process

$$
W:=\left(W_{l, t, v}, v \geq 0\right):=\left(\left(X_{l, t, v}, t-\int_{0}^{v} X_{l, t, u} d u\right), v \geq 0\right)
$$

on the space of continuous E-valued paths. Then $\left(Q^{w}, w \in E\right)$ is the collection of laws of a strong Markov process $W$ with state space $E$ with $(0,0)$ as an absorbing state which is reached in finite time $Q^{w}$ a.s. for all $w \in E$.

The scaling property (iii) implies that in formulating results about $X$ there is no loss of generality in supposing that $t=1$. However, this reduction tends to obscure basic properties of $X$ such as the Markov property (iv) of $W$, where it is essential to involve both $l$ and $t$. Figure 1 displays approximations to trajectories of the process $\left(X_{l, t, v}, v \geq 0\right)$ for $l=0,1,2,3$ and $t=1$, obtained by computer simulation. The main result of the paper is the following theorem, which, together with the well-known formula [48]

$$
P\left(L_{t, 0}^{|\mathrm{br}|} / 2>x\right)=\exp \left(-\frac{x^{2}}{2 t}\right) \text { for } t>0, x \geq 0,
$$

determines the distribution of the process $\left(L_{t, v}^{|\mathrm{br}|}, v \geq 0\right)$. 


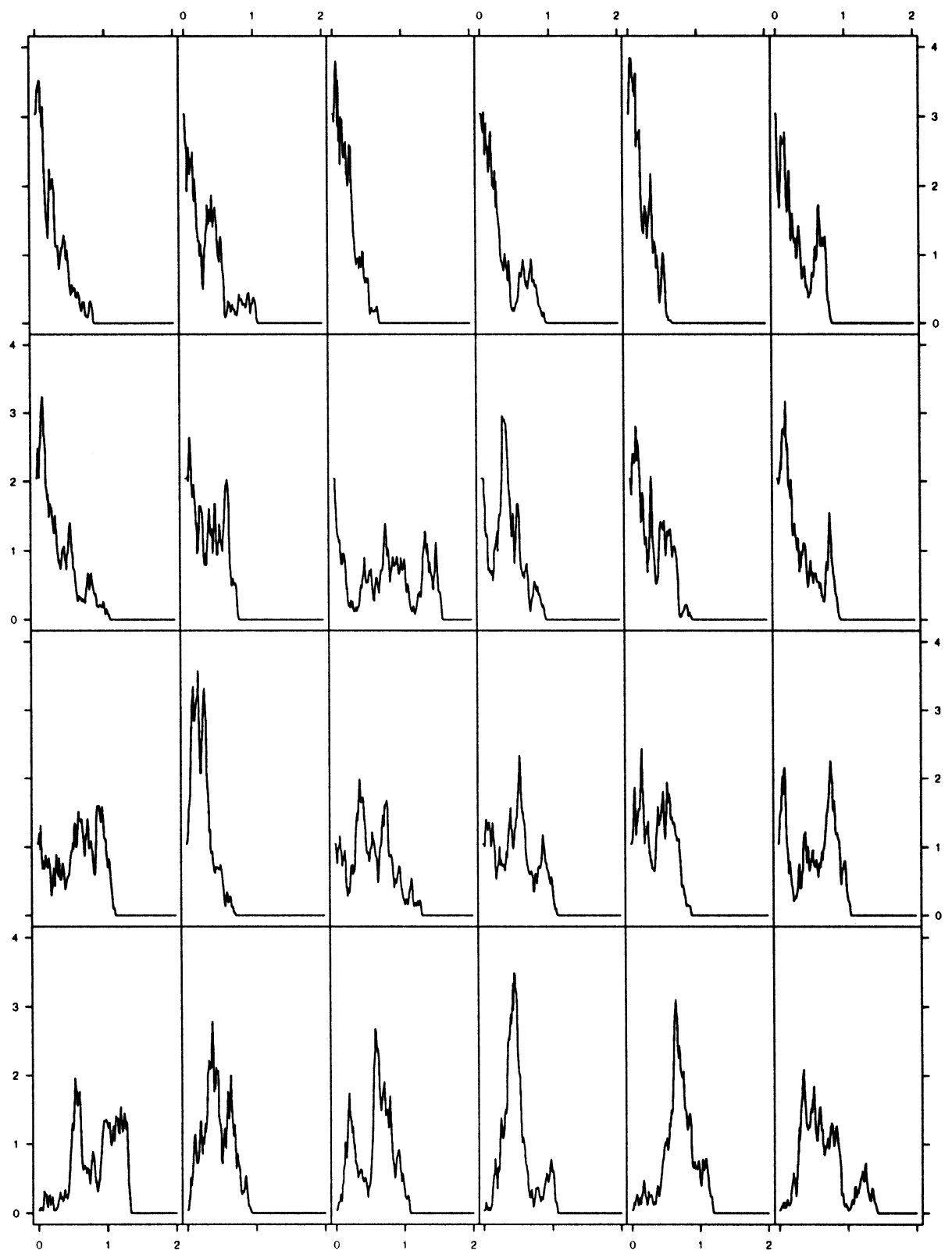

FIG. 1. Simulated trajectories of the local time process of a reflecting Brownian bridge of length 1. Each panel shows an exact computer simulation of the height profile of a uniform random forest of $k$-rooted trees with $n=2500$ vertices, generated from binomial random variables via Lemma 9 and scaled as in (17) to approximate $\left(X_{l, 1, v}, v \geq 0\right)$ governing the local times of a reflecting Brownian bridge of length 1 given local time l at 0 . In each panel, $v$ ranges from 0 to 2 on a horizontal scale, and the vertical scale for local time ranges from 0 to 4 . The area under each trajectory differs negligibly from 1. Each row shows six repetitions for a given initial value $l=2 k / \sqrt{n}=k / 25$, with $l=1 / 25$ in the bottom row approximating the local time process of a Brownian excursion, and $l=1,2,3$ in rows above. 
THEOREM 4. For each $t>0$ the collection of laws of $\left(X_{l, t, v}, v \geq 0\right)$ on the path space $C[0, \infty)$, parameterized by $l \geq 0$, is the unique weakly continuous version of the conditional law of $\left(L_{t, v}^{|\mathrm{br}|}, v \geq 0\right)$ given $\left(L_{t, 0}^{|\mathrm{br}|}=l\right)$,

$$
\left(L_{t, v}^{|\mathrm{br}|}, v \geq 0 \mid L_{t, 0}^{|\mathrm{br}|}=l\right)=_{d}\left(X_{l, t, v}, v \geq 0\right) .
$$

It is intuitively clear, and made rigorous by Lemma 12, that conditioning a RBB of length $t$ to have zero local time at 0 should produce a Brownian excursion of length $t$. Thus the particular case $l=0$ of Theorem 4 yields the following description of the process of local times of a Brownian excursion. That this process solves a SDE of the form (2) is implicit in [27], page 264, and explicit in [30], page 11. However, these authors do not discuss the uniqueness issue settled by Lemma 1 .

COROLlary 5. The process of local times up to time $t$ of a Brownian excursion of length $t$ is identical in law to the process defined by the solution of the $S D E$ (2) for $l=0$,

$$
\left(L_{t, v}\left(B^{\mathrm{ex}, t}\right), v \geq 0\right)={ }_{d}\left(X_{0, t, v}, v \geq 0\right) .
$$

If the path dependent drift $\delta_{v}(X)$ in the SDE (2) is replaced by a constant drift $\delta$, then (2) becomes the SDE governing a $B E S Q_{l}^{(\delta)}$ process $\left(X_{l, v}^{(\delta)}, v \geq 0\right)$, that is a Bessel squared process of dimension $\delta$ started at $l$. See $[71,63,68]$. This process appears for $\delta=0,2$ and 4 in the Ray-Knight description of Brownian local time processes, also for other real $\delta$, both fixed and path dependent, as the distribution of local times of appropriately perturbed Brownian motions. See Section 7 for references to such results. For $\tau_{l}:=$ $\inf \left\{t: L_{t, 0}(|B|)=l\right\}$, the Ray-Knight theorem,

$$
\left(L_{\tau_{l}, v}(|B|), v \geq 0\right)={ }_{d}\left(X_{l, v}^{(0)}, v \geq 0\right),
$$

combined with the well-known and easily rigorized identity in law,

$$
\left(B_{s}^{|\mathrm{br}|, t}, 0 \leq s \leq t \mid L_{t, 0}^{|\mathrm{br}|}=l\right)={ }_{d}\left(\left|B_{s}\right|, 0 \leq s \leq \tau_{l} \mid \tau_{l}=t\right)
$$

for $l, t>0$ shows that Theorem 4 implies the following corollary (obtained independently in [47] Section 4), and vice versa.

LEMMa 6. For all $l>0$ and $t>0$,

$$
\left(X_{l, v}^{(0)}, v \geq 0 \mid \int_{0}^{\infty} X_{l, v}^{(0)} d v=t\right)={ }_{d}\left(X_{l, t, v}, v \geq 0\right),
$$

where the distribution of the right side provides the unique determination of the conditional distribution of the left side that is weakly continuous in $t$.

The rest of this paper is organized as follows. Section 2 shows how the process defined by the SDE (2) arises as the weak limit of a suitably normalized Poisson-Galton-Watson branching process conditioned on its total progeny. The limit process can then be identified with the process 
appearing in Theorem 4. Section 3 explains the connection with random forests and random mappings. Section 4 lays out a series of corollaries which amplify the meaning of Proposition 3 and Theorem 4 in various ways. One of these corollaries is the Biane and Yor description of the process of local times of $B^{\mid \mathrm{br|,1}}$. Another is Jeulin's corresponding description of the process of local times of a Brownian excursion. It will be seen that this line of reasoning can also be reversed to recover Theorem 4 from either of these descriptions. Section 5 deduces from these results some explicit formulas regarding the distribution of local times of the reflecting bridge. Section 6 records a variation of Theorem 4 for an unreflected bridge. Finally, some concluding remarks and further references to related work are made in Section 7.

2. The branching process approximation. According to a result in the theory of branching processes, first indicated by Feller [19] and further developed by Lamperti [40,39] and Lindvall [49], if $Z_{k}(h)$ for $h=0,1,2, \ldots$ denotes the number of individuals in generation $h$ of a Galton-Watson process started with $k$ individuals in which the offspring distribution has mean 1 and finite variance $\sigma^{2}>0$, and $Z_{k}(h)$ is defined for all $h \geq 0$ by linear interpolation between integers, then as $m \rightarrow \infty$ and $k$ varies with $m$ in such a way that $(2 k) /(\sigma m) \rightarrow l$,

$$
\left(\frac{2}{\sigma m} Z_{k}\left(\frac{2 m v}{\sigma}\right), v \geq 0\right) \rightarrow_{d}\left(X_{l, v}^{(0)}, v \geq 0\right)
$$

where $\left(X_{l, v}^{(0)}, v \geq 0\right)$ is the $B E S Q_{l}^{(9)}$ process defined by the SDE

$$
X_{0}=l ; \quad d X_{v}=2 \sqrt{X_{v}} d \beta_{v}
$$

and $\rightarrow_{d}$ is the usual notion of convergence of distributions on $C[0, \infty)$. To check the nonstandard normalizations in (13), observe that if the process on the left side has value $x$ at $v$ such that $2 m v / \sigma$ equals an integer $h$, then $Z_{k}(h)=x \sigma m / 2$. The number $Z_{k}(h+1)$ in the next generation of the branching process therefore has variance $(x \sigma \mathrm{m} / 2) \sigma^{2}$. The increment of the process on the left side over the next $v$-increment of $\sigma /(2 \mathrm{~m})$ has this variance multiplied by $(2 / \sigma \mathrm{m})^{2}$. So along the grid of multiples of $\sigma /(2 \mathrm{~m})$, the variance of increments of the normalized process on the left side per unit $v$-increment, from one grid point to the next, given the normalized process has value $x$ at the first grid point, is

$$
\left(\frac{x \sigma m}{2}\right) \sigma^{2}\left(\frac{2}{\sigma m}\right)^{2}\left(\frac{\sigma}{2 m}\right)^{-1}=4 x=(2 \sqrt{x})^{2}
$$

in accordance with the $B E S Q^{(0)} \operatorname{SDE}$ (14). Kawazu and Watanabe [28] showed that if the branching process is modified by allowing an immigration term, then the weak limit is a $B E S Q_{l}^{(\delta)}$ process with $\delta$ representing an asymptotic rate of immigration per unit time. They showed also that this result for $\delta=0$ and $\delta=2$ yields the basic Ray-Knight theorems when applied to the branching processes with geometric offspring distribution 
derived from upcrossings of the Brownian path. See also [41] for another exposition of this idea and [10,11] and [33] for more about approximation of Brownian local times using random walks.

Consider now the distribution of the process $\left(Z_{k, n}(h), h \geq 0\right)$ defined by conditioning $\left(Z_{k}(h), h \geq 0\right)$ on the event that its total progeny $\sum_{h=0}^{\infty} Z_{k}(h)$ equals $n$. So

$$
\left(Z_{k, n}(h), h \geq 0\right)={ }_{d}\left(Z_{k}(h), h \geq 0 \mid \sum_{h=0}^{\infty} Z_{k}(h)=n\right),
$$

where it is assumed now that the offspring distribution is aperiodic, so the conditioning event has strictly positive probability for all sufficiently large $n$. In view of (13) for $m=\sqrt{n}$, in an asymptotic regime with $n \rightarrow \infty$ and $(2 k) /(\sigma \sqrt{n}) \rightarrow l$

$$
\frac{1}{n} \sum_{h=0}^{\infty} Z_{k}(h) \approx \int_{0}^{\infty} \frac{2}{\sigma \sqrt{n}} Z_{k}(2 \sqrt{n} v / \sigma) d v
$$

in the sense that the difference converges in probability to 0 as $n \rightarrow \infty$. So one expects that as $n \rightarrow \infty$ and $(2 k) /(\sigma \sqrt{n}) \rightarrow l$ for some $l \geq 0$,

$$
\left(\frac{2}{\sigma \sqrt{n}} Z_{k, n}(2 \sqrt{n} v / \sigma), v \geq 0\right) \rightarrow_{d}\left(X_{l, 1, v}, v \geq 0\right),
$$

where $\left(X_{l, 1, v}, v \geq 0\right)$ for $l>0$ is identified via (12) as the limit process in (13) conditioned to have integral equal to 1 . If $l=0$, this line of reasoning fails because the limit process in (13) is identically equal to 0 . However, [16] and [30] show that (17) holds in this case with the law of the limiting process $\left(X_{0,1, v}, v \geq 0\right)$ identified as that of the process of local times of a Brownian excursion. It appears that (17) can be justified in general by some combination of arguments from the present paper and from [30], but that will not be attempted here. See also [31] regarding the case when the offspring distribution has infinite variance.

In view of the scaling properties of the processes involved, it is clear that Corollary 6 can be deduced from the combination of (13) and (17) for any particular choice of offspring distribution for the Galton-Watson process. Then Theorem 4 can be deduced from Corollary 6 via the Ray-Knight theorem (11), as indicated in the introduction. Thus Theorem 4 is a consequence of the following result.

THEOREM 7. The convergence in distribution (17) holds with $\sigma=1$ as $n \rightarrow \infty$ and $(2 k) / \sqrt{n} \rightarrow l \geq 0$, for $\left(Z_{k, n}(h), h \geq 0\right)$ a Galton-Watson process with Poisson offspring distribution started with $k$ individuals and conditioned to have total progeny $n$ and with the limit process $\left(X_{l, 1, v}, v \geq 0\right)$ constructed as in Lemma 1 for $t=1$.

The proof of Theorem 7 is based on the following lemma. Note that if $Z_{k, n}(h)$ is interpreted as the number of vertices at level $h$ in a forest with $n$ 
vertices defined by a collection of $k$ family trees, one for each initial individual in the Galton-Watson process, then for $h=0,1, \ldots$, the random variable

$$
A_{k, n}(h):=n-\sum_{i=0}^{h} Z_{k, n}(i)
$$

represents the number of vertices in the forest strictly above level $h$.

LEMMA 8. Let $X_{1}, X_{2}, \ldots$ be a sequence of independent random variables with some distribution $p$ on $\{0,1,2, \ldots\}$, and set $S_{j}=X_{1}+\cdots+X_{j}$. Fix $1 \leq$ $k<n$ with $P\left(S_{n}=n-k\right)>0$. Let $\left(Z_{k, n}(h), h=0,1,2, \ldots\right)$ be a GaltonWatson branching process with offspring distribution $p$ started with $k$ individuals and conditioned to have total progeny $n$. Let $Z(h), h=0,1, \ldots$ be a sequence of nonnegative integer random variables and set

Then

$$
A(h):=n-\sum_{i=0}^{h} Z(i) \text { and } W(h):=(Z(h), A(h)) .
$$

$$
(Z(h), h=0,1,2, \ldots)={ }_{d}\left(Z_{k, n}(h), h=0,1,2, \ldots\right)
$$

if and only if the sequence $(W(h), h=0,1,2, \ldots)$ is a Markov chain with state space

$$
E^{\prime}:=(\{1,2, \ldots,\} \times\{0,1,2, \ldots,\}) \cup\{(0,0)\},
$$

initial state $(k, n-k)$, and the following stationary transition probabilities: for all $h=0,1,2, \ldots$ and all $(z, a) \in E^{\prime}$ with $a>0$ and $\left(z_{1}, a_{1}\right) \in E^{\prime}$ with $z_{1}+a_{1}=a \geq 1$,

$$
\begin{aligned}
P(W(h+1) & \left.=\left(z_{1}, a_{1}\right) \mid W(h)=(z, a)\right) \\
& =\frac{z_{1}(z+a)}{z a} P\left(S_{z}=z_{1} \mid S_{z+a}=a\right),
\end{aligned}
$$

whereas $P(W(h+1)=(0,0) \mid W(h)=(z, 0))=1$, and all other transition probabilities are zero.

Proof. This is easily verified by a computation using the well-known formula [18] for the distribution of the total progeny in the branching process starting with $k$ individuals,

$$
P\left(\sum_{h=0}^{\infty} Z_{k}(h)=n\right)=\frac{k}{n} P\left(S_{n}=n-k\right)
$$

together with the Markov property of the branching process, and Bayes rule.

See [62] for a recent review of the fundamental formula (21) and its various probabilistic and combinatorial equivalents. As a check on formula (20), one that the sum of probabilities in (20) over all $0 \leq z_{1} \leq a$ is 1 , due to the well-known formula

$$
E\left(S_{z} \mid S_{z+a}=a\right)=\frac{z a}{(z+a)}
$$


which follows from exchangeability of the $X_{i}$. According to (20), given $W_{k, n}(h)=(z, a)$, the distribution of $Z_{k, n}(h+1)$ is obtained by size-biasing of the distribution of $S_{z}$ given $S_{z+a}=a$, while $A_{k, n}(h+1)=a-Z_{k, n}(h+1)$. For a Poisson offspring distribution, the law of $S_{z}$ given $S_{z+a}=a$ is bino$\operatorname{mial}(a, z /(z+a))$. It is elementary that a size-biased binomial $(n, p)$ variable is 1 plus a binomial $(n-1, p)$ variable, so the previous lemma can be specialized as follows.

Lemma 9. Fix $1 \leq k<n$. A sequence $(Z(h), h=0,1, \ldots)$ has the same distribution as a Galton-Watson process with a Poisson offspring distribution started with $k$ individuals and conditioned on total progeny equal to $n$, if and only if the sequence evolves by the following mechanism: $Z(0)=k$ and for each $h=0,1, \ldots$,

$$
\begin{aligned}
(Z(h+1) \mid Z(i), 0 & \leq i<h, Z(h)=z, A(h)=a) \\
& ={ }_{d} 1+\operatorname{bin}\left(a-1, \frac{z}{a+z}\right),
\end{aligned}
$$

where $A(h):=n-\sum_{i=0}^{h} Z(i)$ and $\operatorname{bin}(m, p)$ is a binomial random variable with parameters $m$ and $p$, with the conventions $\operatorname{bin}(-1, p)=-1$ and $\operatorname{bin}(0, p)=0$.

Proof of Theorem 7. Consider the rescaled process on the left side of (17) in the Poisson case, so $\sigma=1$, in an asymptotic regime with $n \rightarrow \infty$ and $2 k / \sqrt{n} \rightarrow l$ for some $l \geq 0$. From (22), in the limit as $n, z$ and $a$ tend to $\infty$ with $2 z / \sqrt{n} \rightarrow x$ and $a / n \rightarrow p$, for integer $h$ the increment $\Delta_{k, n}(h):=$ $Z_{k, n}(h+1)-Z_{k, n}(h)$ is such that the corresponding normalized increment $\Delta_{k, n}^{*}(h):=2 \Delta_{k, n}(h) / \sqrt{n}$ has the following conditional mean and variance given a history $\left(Z_{k, n}(i), 0 \leq i \leq h\right)$ with $W_{k, n}(h)=(z, a)$ :

$$
\begin{aligned}
E\left(\Delta_{k, n}^{*}(h) \mid W_{k, n}(h)\right. & =(z, a))=\frac{2}{\sqrt{n}}\left(1+\frac{(a-1) z}{a+z}-z\right) \\
& \approx\left(4-\frac{x^{2}}{p}\right) \frac{1}{2 \sqrt{n}}, \\
\operatorname{Var}\left(\Delta_{k, n}^{*}(h) \mid W_{k, n}(h)\right. & =(z, a))=\frac{4}{n} \frac{(a-1) z a}{(a+z)^{2}} \approx 4 x \frac{1}{2 \sqrt{n}} .
\end{aligned}
$$

The relative errors of approximation here are negligible as $n \rightarrow \infty$, uniformly in $h$, provided $x<1 / \varepsilon$ and $p>\varepsilon$, which can be arranged by a localization argument, stopping the normalized process when either its value exceeds $x$ or its integral exceeds $1-p$. Since $\Delta_{k, n}^{*}(h)$ is the increment of the normalized process over a time interval of length $1 /(2 \sqrt{n})$, and the value of $p \approx A_{k, n}(h) / n$ can be recovered from the path of the normalized process with a negligible error via

$$
p \approx \frac{A_{k, n}(h)}{n}=1-\frac{1}{n} \sum_{i=0}^{h} Z_{k, n}(i) \approx 1-\int_{0}^{h /(2 \sqrt{n})} \frac{2}{\sqrt{n}} Z_{k, n}(2 \sqrt{n} v) d v
$$


these calculations show that the normalized process is governed asymptotically by the SDE (2) and (3). According to Lemma 1, the SDE has a unique strong solution, so the conclusion follows by application of known results regarding the weak convergence of a sequence of Markov chains to the solution of an SDE [38, 37]. See in particular [37] Theorem 5.4, regarding unbounded coefficients.

3. Application to forests and mappings. It is known $[36,1]$ that each of the two processes $\left(Z_{k, n}(h), h \geq 0\right)$ defined in (1) and (2) below has the distribution of a Galton-Watson branching process with Poisson offspring distribution started with $k$ individuals and conditioned to have total progeny $n$. See [62] for a quick proof of this fact in case (1). This case can also be deduced by using classical enumerations of trees and forests, reviewed in [61] to show the conditions of Lemma 9 are satisfied.

1. In a random forest with uniform distribution on the set of all rooted forests of $k$ trees labeled the set $[n]:=\{1, \ldots, n\}$, let $Z_{k, n}(h)$ equal the number of vertices at height $h$ above the roots. Call this process $\left(Z_{k, n}(h), h \geq 0\right)$ the height profile of the forest.

2. For $M$ a mapping from [n] to [n], with iterates $M^{m}$ for $m=0,1,2, \ldots$, call $v \in[n]$ a cyclic point of $M$ if $M^{m}(v)=v$ for some $m>0$. Let $\operatorname{cyclic}(M)$ be the set of cyclic points of $M$. For $v \in[n]$ let $h(v, M)$ be the least $m \geq 0$ such that $M^{m}(v) \in \operatorname{cyclic}(M)$. So $h(v, M)$ is the height of $v$ in the usual forest derived from $M$ whose set of roots is cyclic $(M)$. For $h=0,1,2, \ldots$, let $Z_{*, n}(h)$ be the number of $v \in[n]$ such that $h\left(v, M_{n}\right)=$ $h$, for $M_{n}$ a random mapping from $[n]$ to $[n]$, with uniform distribution on the set of $n^{n}$ such mappings, as studied in [36, 1]. Call this process $\left(Z_{*, n}(h), h \geq 0\right)$ the height profile of the mapping forest. Let $\left(Z_{k, n}(h)\right.$, $h \geq 0$ ) be the height profile of the mapping forest conditioned on the event $\left(Z_{*, n}(0)=k\right)$ that $M_{n}$ has exactly $k$ cyclic points.

That $\left(Z_{k, n}(h), h \geq 0\right)$ has the same distribution in (2) as in (1) is evident because given that $M_{n}$ has $k$ cyclic points, the forest generated by $M_{n}$ is a uniform random forest of $k$ rooted trees labeled by [ $n$ ], exactly as supposed in (1). Theorem 7 now yields the corollary.

CoRollary 10. If $\left(Z_{k, n}(h), h \geq 0\right)$ is either:

(i) The height profile of a uniform random forest of $k$ rooted trees labeled by $[n]$, or

(ii) the height profile of the forest derived from a random mapping from $[n]$ to $[n]$ conditioned to have $k$ cyclic points,

then the distribution of the sequence $\left(Z_{k, n}(h), h \geq 0\right)$ is that described by Lemma 9, and in the limit regime as $n \rightarrow \infty$ and $2 k / \sqrt{n} \rightarrow l \geq 0$,

$$
\left(\frac{2}{\sqrt{n}} Z_{k, n}(2 \sqrt{n} v), v \geq 0\right) \rightarrow_{d}\left(X_{l, 1, v}, v \geq 0\right) \text {. }
$$


Aldous-Pitman [1] showed how a uniform random mapping $M_{n}$ can be recoded as a nonuniformly distributed random walk of $2 n$ steps starting and ending at 0 , with each tree component of the forest generated by $M_{n}$ corresponding to an excursion of the walk away from 0 , in such a way that as $n \rightarrow \infty$ the normalized walk converges in distribution to a reflecting Brownian bridge of length 1 . The following further corollary is now obtained by mixing the result of the previous corollary with respect to the distribution of the number $Z_{*, n}(0)$ of cyclic points of $M_{n}$. It is well known [1] that for all $x>0$,

$$
\lim _{n \rightarrow \infty} P\left(Z_{*, n} / \sqrt{n}>x\right)=\exp \left(-x^{2} / 2\right)
$$

So Theorem 4 and Corollary 10 combine with (25) and (8) to yield the Corollary.

CoROLlaRy 11 (Drmota-Gittenberger [17]). The normalized height profile of the forest derived from a uniform random mapping $M_{n}$ converges weakly to the process of local times of a reflecting Brownian bridge of length 1,

$$
\left(\frac{2}{\sqrt{n}} Z_{*, n}(2 \sqrt{n} v), v \geq 0\right) \rightarrow_{d}\left(L_{1, v}^{|\mathrm{br}|}, v \geq 0\right) .
$$

A second proof of Theorem 4 can be given by comparison of Corollary 10 with the known result (26) or with the weaker integrated form of (26) given in [1].

4. Related results. This section presents a series of results related to Theorem 4. Those deduced from the theorem are labeled as corollaries, while those proved independently of the theorem are labeled lemmas. In particular, the Biane and Yor description of $\left(L_{t, v}^{|\mathrm{br}|}, v \geq 0\right)$ is obtained as a corollary. This description combined with the lemmas yields a third proof of Theorem 4 .

Observe first that in terms of the local time representation of $X$ provided by Theorem 4, the Markov property of the process $W$ described in Proposition 3 , which is the continuous analog of the Markov property of $W$ in Lemma 8 , amounts to the following equality of distributions on $C[0, \infty)$, where $\operatorname{dist}(X \mid Y)$ stands for the conditional distribution of $X$ given $Y$ : for all $t>r>0, l \geq 0$, $v \geq 0$,

$$
\begin{aligned}
& \operatorname{dist}\left(L_{t, v+z}^{|\mathrm{br}|}, z \geq 0 \mid L_{t, u}^{|\mathrm{br}|}, 0 \leq u \leq v \text { with } L_{t, v}^{|\mathrm{br}|}=l, t-\int_{0}^{v} L_{t, u}^{|\mathrm{br}|} d u=r\right) \\
& =\operatorname{dist}\left(L_{r, z}^{|\mathrm{br}|}, z \geq 0 \mid L_{r, 0}^{|\mathrm{br}|}=l\right) .
\end{aligned}
$$

Lemma 12 prepares for a refinement of this identity in law which is stated in Lemma 13.

LEMMA 12. For each $t>0$, there exists on the path space $C[0, t]$ a unique family of conditional distributions for $\left(B_{s}^{|\mathrm{br}|, t}, 0 \leq s \leq t\right)$ given $L_{t, 0}^{|\mathrm{br}|}=l$, say 
$\left(P^{l, t}, l \geq 0\right)$, that is weakly continuous in $l$. In particular, the law $P^{0, t}$ is the law of a Brownian excursion of length $t$.

Proof. The existence of such a continuous family $\left(P^{l, t}, l \geq 0\right)$ follows from the construction of the RBB by first constructing its zero set, then piecing together independent Brownian excursions over the maximal open intervals in the complement of the zero set. See [65] for an explicit description of the law of the ranked lengths of the complementary intervals given $L_{t, 0}^{|\mathrm{br}|}=l$. Given the lengths, each interval is assigned an independent local time value with uniform distribution on $[0, l]$, and then the lengths are laid down in the order of the local time variables. It follows from this description that for each $t>0$ and $\varepsilon>0$ there exists $\delta$ such that for $l<\delta$, with $P^{l, t}$ probability at least $1-\varepsilon$, there is a complementary interval of length at least $t-\varepsilon$. This implies easily that $P^{0, t}$ is the law of a Brownian excursion of length $t$.

This construction of a Brownian excursion of length $t$ by conditioning $B^{|\mathrm{br}|, t}$ on $L_{t, 0}^{|\mathrm{br}|}=0$ parallels similar constructions by conditioning a Brownian bridge $B^{\text {br, } t}$ of length $t$ on $Z_{t}=0$ for suitable $Z_{t}$, due to [9] for $Z_{t}:=$ $\inf _{0 \leq s \leq t} B_{s}^{\mathrm{br}, t}$ and [14] for $Z_{t}:=\int_{0}^{t} 1\left(B_{s}^{\mathrm{br}, t} \leq 0\right) d s$.

If $B^{|\mathrm{br}|, l, t}$ denotes a process with law $P^{l, t}$, then Lemma 12 allows Theorem 4 to be recast as

$$
\left(L_{t, v}\left(B^{|\mathrm{br}|, l, t}\right), v \geq 0\right)={ }_{d}\left(X_{l, t, v}, v \geq 0\right) .
$$

For $l=0$, Corollary 5 is then recovered from Lemma 12 . The law $P^{l, t}$ could also be constructed as in [1] as a weak limit from a uniform mapping $M_{n}$ conditioned to have around $k=l \sqrt{n} / 2$ cyclic points, or from a random rooted forest of $k$ trees with $n$ vertices, or by similar conditioning of a uniform lattice walk path of length $2 n$ on its number of returns to 0 .

Lemma 13. Fix $t>0$. For $v \geq 0$ let $Y^{v,-}$ denote the process with lifetime

$$
\zeta^{v,-}:=\int_{0}^{t} 1\left(B_{s}^{|\mathrm{br}|, t} \leq v\right) d s=\int_{0}^{v} L_{t, u}^{|\mathrm{br}|} d u
$$

defined by deleting the excursions of $B^{|\mathrm{br}|, t}$ above $v$ and closing up the gaps, and let $Y^{v,+}$ denote the process with lifetime

$$
\zeta^{v,+}:=\int_{0}^{t} 1\left(B_{s}^{|b r|, t}>v\right) d s=t-\int_{0}^{v} L_{t, u}^{|b r|} d u
$$

defined by deleting all portions of the path of $B^{|\mathrm{br}|, t}$ below $v$, closing up the gaps and finally subtracting $v$ so the path starts and ends at 0 . Then:

(i) The process $\left(L_{t, u}^{\mid b r}, 0 \leq u \leq v\right)$ is the restriction to $[0, v]$ of the process of local times of $Y^{v,-}$ up to time $\zeta^{v,-}$.

(ii) The process ( $L_{t, v+z}^{|\mathrm{br}|}, z \geq 0$ ) is the process of local times at levels $z$ of the process $Y^{v,+}$ up to time $\zeta^{v,+}$. 
(iii) For $l^{\prime} \geq 0, r>0$,

$$
\operatorname{dist}\left(Y^{v,+} \mid Y^{v,-} \text { with } L_{t, v}^{|\mathrm{br}|}=l^{\prime}, \zeta^{v,+}=r\right)=P^{r, l^{\prime}} .
$$

(iv) These results hold also for each $l \geq 0$ with the reflecting bridge $B^{|\mathrm{br}|, t}$ replaced by a bridge $B^{|\mathrm{br}|, l, t}$ with the law $P^{l, t}$ described in Lemma 12 , and in particular for $l=0$ with $B^{|\mathrm{br}|, t}$ replaced by $B^{\mathrm{ex}, t}$, an excursion of length $t$.

Proof. See [25], Section 2.11, for details of the construction involved in the definition of the processes $Y^{v,-}$ and $Y^{v,+}$. Properties (i) and (ii) follow immediately from the construction. Property (iii) can be deduced from the structure of Brownian excursions and excursion filtration exposed in [76, 24, 50, 69, 70]. Property (iv) then follows from (iii) and the definition of the conditioned bridge laws $P^{l, t}$.

Consider now the family of laws $B E S_{x}^{(3)}, x \geq 0$ of a three-dimensional Bessel process which may be constructed as the solution of the SDE

$$
R_{0}=x ; \quad d R_{t}=R_{t}^{-1} d t+d \beta_{t}
$$

for a Brownian motion $\beta$. For $t>0$ let $R^{x, y, t}$ denote a $B E S^{(3)}$ bridge from $x$ to $y$ of length $t$, that is a $B E S_{x}^{(3)}$ process $R$ conditioned on $R_{t}=y$, regarded as process parameterized by $[0, t]$. It is easily seen that such a process $R^{x, y, t}$ may be constructed for $0 \leq s \leq t$ by the formula

$$
R_{s}^{x, y, t}:=\sqrt{\left(x+(y-x) s / t+B_{1, s}^{\mathrm{br}, t}\right)^{2}+\left(B_{2, s}^{\mathrm{br}, t}\right)^{2}+\left(B_{3, s}^{\mathrm{br}, t}\right)^{2}},
$$

where the $\left(B_{i, s}^{\mathrm{br}, t}, 0 \leq s \leq t\right)$ for $i=1,2,3$ are three independent copies of a one-dimensional Brownian bridge of length $t$. As a consequence of this description and Itô's formula, $R^{x, y, t}$ can also be constructed as the solution over $[0, t]$ of the SDE

$$
R_{0}=x ; \quad d R_{s}=\left(\frac{1}{R_{s}}+\frac{\left(y-R_{s}\right)}{(t-s)}\right) d s+d \gamma_{s}
$$

for a Brownian motion $\gamma$. See also [78, 59, 22, 68] for background. The following lemma was suggested by the results of [27] and [7] presented in Corollary 16.

LEMMA 14. For $l \geq 0, t>0$ let $R^{l, 0, t}$ be the process derived from $\left(X_{l, t, v}\right.$, $v \geq 0$ ) via the formula

$$
2 R_{s}^{l, 0, t}:=X_{l, t, v} \text { for the least } v: \int_{0}^{v} X_{l, t, u} d u=s \quad \text { where } 0 \leq s \leq t .
$$

Then $R^{l, 0, t}$ is a $B E S^{(3)}$ bridge from $l$ to 0 of length $t$, and $\left(X_{l, t, v}, v \geq 0\right)$ can be recovered from $R^{l, 0, t}$ via the formula

$$
X_{l, t, v}=2 R_{s}^{l, 0, t} \text { for the least } s: \int_{0}^{s} \frac{d r}{2 R_{r}^{l, 0, t}}=v
$$


Consequently, starting from any $B E S^{(3)}$ bridge $R^{l, 0, t}$ from $l$ to 0 of length $t$, the process $X$ defined by (31) has the same distribution as $X$ defined by the $S D E(2)$.

PRoof. The recipe (31) for inverting the time change (30) is easily checked, so it suffices to show that if $R:=\left(R_{s}^{l, 0, t}, 0 \leq s \leq t\right)$ solves the SDE (29), for $(x, y)=(l, 0)$; that is

$$
d R_{s}=\left(\frac{1}{R_{s}}-\frac{R_{s}}{(t-s)}\right) d s+d \gamma_{s}
$$

for some Brownian motion $\gamma$, then $X:=\left(X_{l, t, v}, v \geq 0\right)$ defined by (31) solves the SDE (2) for some Brownian motion $\beta$. But from (30) and (31),

$$
d X_{v}=2 d R_{s} \quad \text { where } s=\int_{0}^{v} X_{u} d u .
$$

A level increment $d v$ for $X$ corresponds to a time increment $d s=X_{v} d v$ for $R$, and $R_{s}=X_{v} / 2$, so

$$
d X_{v}=2\left(\frac{1}{X_{v} / 2}-\frac{X_{v} / 2}{\left(t-\int_{0}^{v} X_{u} d u\right)}\right) X_{v} d v+2 \sqrt{X_{v}} d \beta_{v}
$$

for some other Brownian motion $\beta$, where the factor $\sqrt{X_{v}}$ appears in the diffusion term due to Brownian scaling, and (32) simplifies to (2). As a technical point, the definition of $\beta$ above the level $\int_{0}^{t} d r / 2 R_{r}^{l, 0, t}$ when $X$ hits 0 may require enlargement of the probability space. See [68], Chapter V, for a rigorous discussion of such issues.

Lemma 15. The laws of a Brownian excursion $B^{\mathrm{ex}, t}$ and a Brownian meander $B^{\mathrm{me}, t}$, each of length $t$, can be expressed as follows in terms of the laws of $B E S^{(3)}$ bridges $R^{x, y, t}$ :

(i) $[77]$.

$$
B^{\mathrm{ex}, t}={ }_{d} R^{0,0, t}
$$

(ii) [22]. The final value $B_{t}^{\mathrm{me}, t}$ of the meander has the distribution of $\sqrt{t} R$ for $R$ with the standard Rayleigh distribution $P(R>r)=\exp \left(-r^{2} / 2\right), r>0$, and

$$
\left(B^{\mathrm{me}, t} \mid B_{t}^{\mathrm{me}, t}=y\right)={ }_{d} R^{0, y, t} .
$$

The above results now combine easily to yield the corollary.

Corollary 16. For a process $Y:=\left(Y_{s}, 0 \leq s \leq t\right)$ admitting a local time process $\left(L_{t, v}(Y), v \geq 0\right)$, define a process $\hat{L}(Y):=\hat{L}_{r}(Y), 0 \leq r \leq t$ by $\hat{L}_{r}(Y):=L_{t, v(r)}(Y)$ where $v(r):=\sup \left\{y \geq 0: \int_{y}^{\infty} L_{t, x} d x>r\right\}$. So $\hat{L}_{r}(Y)$ is the local time of $Y$ at a level $v(r)$ above which $Y$ spends time $r$. $\hat{L}(Y) / 2$.

(i) ([27], page 264) If $Y$ is a Brownian excursion of length $t$, then so is 
(ii) ([7], Theorem (5.3)) If $Y$ is a reflecting Brownian bridge of length $t$, then $\hat{L}(Y) / 2$ is a Brownian meander of length $t$.

(iii) If $Y$ has the law $P^{t, l}$ of $B^{|\mathrm{br}|, t}$ given $L_{t, 0}^{|\mathrm{br}|}=l$, then $\hat{L}(Y) / 2$ is a $B E S^{(3)}$ bridge from 0 to $l$ of length $t$.

Indeed, by combining (28) and Lemma 14, for $Y$ as in (iii), the process $\hat{L}(Y) / 2$ is seen to be a time-reversed copy of $R^{l, 0, t}$, that is, a copy of $R^{0, l, t}$ by a well-known property of one-dimensional diffusion bridges. Now (i) is seen to be the special case $l=0$ of (iii) by Lemma 12 and (33), while (ii) is an integrated form of (iii) by (34) and the fact that $L_{t, 0}^{|\mathrm{br}|} / 2$ and $B_{t}^{\mathrm{me}, t}$ have the same distribution. In view of Lemma 12, the Biane-Yor result (ii) can be disintegrated by conditioning on the local time of $Y$ at 0 to recover (iii). Theorem 4 can then be deduced from (iii) by retracing the above argument via Lemmas 15 and 14 . Theorem 4 can even be deduced from the special case (i) of (iii). For (i) implies the special case $(l=0, t=1)$ of Theorem 4 by the argument just indicated, hence the case $(l=0, t>0)$ by Brownian scaling. By application of Lemma 13 it is clear that the bivariate process $W^{*}$ derived from the local time representation of a nonnegative process $Y$ of length $t$, say,

$$
W^{*}:=\left(\left(L_{t, v}(Y), t-\int_{0}^{v} L_{t, w}(Y) d w\right), v \geq 0\right)
$$

is Markovian with the same transition probabilities whenever $Y$ has the distribution $P^{l, t}$ for any $l \geq 0, t>0$. Denote this process $W^{*}$ by $\left(W_{l, t, v}^{*}\right.$, $v \geq 0$ ). For $Y$ on excursion of length $t$, corresponding to $l=0$, for each $v \geq 0$ the distribution of $W_{0, t, v}^{*}$ has a strictly positive density over $(0, \infty) \times(0, t)$ as well as an atom at the absorbing state $(0,0)$. Due to the Markov property of $W^{*}$, the transition mechanism of this process starting at any state $(l, s)$ with $l>0$ and $s<t$ is therefore determined by the evolution of $W^{*}$ starting in state $(0, t)$ corresponding to an excursion $Y$. But by inspection of the SDE in the excursion case, the same SDE must be solved starting in an arbitrary state $(l, s)$ with $l>0$ and $s<t$. Since $t$ was arbitrary, the conclusion of Theorem 4 follows.

5. Some explicit formulas. Previous results combined with existing results in the literature yield a number of explicit formulas regarding the distribution of the process of local times $\left(L_{1, v}^{|\mathrm{br}|}, v \geq 0\right)$ of a reflecting Brownian bridge of length 1 . As a consequence of the Biane-Yor result of Corollary 16(ii),

$$
\sup _{v \geq 0} L_{1, v}^{|\mathrm{br}|}={ }_{d} 2 \sup _{0 \leq u \leq 1} B_{u}^{\mathrm{me}}={ }_{d} 4 \sup _{0 \leq u \leq 1} B_{u}^{|\mathrm{br}|, 1},
$$

where $B^{\text {me }}$ is a Brownian meander of length 1 , the second equality is due to [29] and the distribution of $\sup _{0 \leq u \leq 1} B_{u}^{|b r|, 1}$ is given by the well-known Kolmogorov-Smirnov formula. Also by Corollary 16(ii),

$$
\left(L_{1,0}^{|\mathrm{br}|}, \sup _{v \geq 0} L_{1, v}^{|\mathrm{br}|}\right)={ }_{d} 2\left(B_{1}^{\mathrm{me}}, \sup _{0 \leq u \leq 1} B_{u}^{\mathrm{me}}\right) .
$$


The joint density of this distribution can be read from known results for the Brownian meander [23]. By conditioning (36) on $L_{1,0}^{|\mathrm{br}|}$, or by Lemma 14,

$$
\left(\sup _{v \geq 0} L_{1, v}^{|\mathrm{br}|} \mid L_{1,0}^{|\mathrm{br}|}=l\right)={ }_{d} \sup _{v \geq 0} X_{l, 1, v}={ }_{d} 2 \sup _{0 \leq u \leq 1} R_{u}^{0, l, 1},
$$

where $R^{0, l, 1}$ is a three-dimensional Bessel bridge from 0 to $l$ of length 1 . The density of this conditional distribution can be read either from the joint density in (36), or from the general formula in [32] for the distribution of the maximum of a $d$-dimensional Bessel bridge.

For fixed $v$, the distribution of

$$
\left(L_{1, v}^{|\mathrm{br}|} \mid L_{1,0}^{|\mathrm{br}|}=l\right)={ }_{d} X_{l, 1, v}
$$

can be evaluated by Theorem 7 as the limit distribution of $2 Z_{k, n}(2 v \sqrt{n}) / \sqrt{n}$ as $n \rightarrow \infty$, with $2 k / \sqrt{n} \rightarrow l$ for $Z_{k, n}(h)$ as in Lemma 9 the number of vertices at level $h$ in a uniform random forest of $k$ rooted trees labeled by [ $n]$. A formula for the density of this limit distribution was found by Pavlov [54], Theorem 6 , in terms of an integral with respect to a two-dimensional probability distribution with an explicit Fourier transform: For $l=0$ more explicit formulas are known from the representation (10) of $X_{0,1, v}$ as the distribution of local time of an excursion at level $v$. See [16] for a review of various representations of this distribution and transform expression for the higher dimensional distributions. Drmota and Gittenberger [17] give similar transforms for the finite-dimensional distributions of $\left(L_{1, v}^{|\mathrm{br}|}, v \geq 0\right)$. Presumably similar transforms can be given for the finite-dimensional distributions of $\left(X_{l, 1, v}, v \geq 0\right.$ ). Pavlov [55], Theorem 2, found a transform for the asymptotic distribution of the maximum height in a random forest of plane rooted trees, which with appropriate scaling can be interpreted via Theorem 7 or geometric offspring distribution as the distribution of

$$
\left(\sup _{0 \leq u \leq 1} B_{u}^{|\mathrm{br}|, 1} \mid L_{1,0}^{|\mathrm{br}|}=l\right)={ }_{d} \inf \left\{v>0: X_{l, 1, v}=0\right\}={ }_{d} \frac{1}{2} \int_{0}^{1} \frac{d u}{R_{u}^{0, l, 1}}
$$

This distribution does not seem to have been studied in the Brownian literature, except in the case $l=0$, when it reduces to the distribution of the maximum of Brownian excursion [15, 29, 7, 3].

See [35] for applications of results of this paper to the computation of moments of the area under a RBB conditional on its local time at zero.

6. The local time process of an unreflected Brownian bridge. Let

$$
L_{t, v}^{\mathrm{br}}:=L_{t, v}\left(B^{\mathrm{br}, t}\right)
$$

denote the local time up to time $t$ at level $v$ of an unreflected Brownian bridge of length $t$. In principle, the law of the process $\left(L_{t, v}^{\mathrm{br}}, v \in \mathbb{R}\right)$ is determined by Ray's [67] description for each $\theta>0$ of the process of local times $\left(L_{T_{\theta}, v}(B), v \in \mathbb{R}\right)$ for $T_{\theta}$ an exponential variable with rate $\theta^{2} / 2$ independent of $B$. According to that description, which is reviewed from a modern perspective in [8], conditionally, given $B_{T_{\theta}}=0$ and $L_{T_{\theta}, 0}(B)=l$, the pro- 
cesses $\left(L_{T_{\theta}, v}(B), v \geq 0\right)$ and $\left(L_{T_{\theta},-v}(B), v \geq 0\right)$ are independent copies of the time-homogeneous diffusion process $Y=\left(Y_{l, \theta, v}, v \geq 0\right)$ defined as the solution of

$$
Y_{0}=l, \quad d Y_{v}=-2 \theta Y_{v} d v+2 \sqrt{Y_{v}} d \beta_{v}
$$

for a Brownian motion $\beta$. Effectively, this describes the distribution of local times of a Brownian bridge of random length with distribution that of $T_{\theta}$ given $B_{T_{\theta}}=0$, which is easily seen to be the $\operatorname{gamma}\left(1 / 2, \theta^{2} / 2\right)$ distribution. By application of Brownian scaling, it is clear that the law of $\left(L_{1, v}^{\mathrm{br}}, v \in \mathbb{R}\right)$, hence that of $\left(L_{t, v}^{\mathrm{br}}, v \in \mathbb{R}\right)$ for each $t>0$, is determined by this description of the law of $\left(L_{T_{\theta}, v}(B), v \in \mathbb{R} \mid B_{T_{\theta}}=0\right)$ even for $\theta=1$. However, it is not easy to use this description to deduce more explicit descriptions of the finitedimensional distributions of $\left(L_{1, v}^{\mathrm{br}}, v \in \mathbb{R}\right)$. For instance, it would be painful to recover from Ray's result the formula

$$
P\left(L_{1, v}^{\mathrm{br}}>x\right)=\exp \left(-2(|v|+x)^{2} / 2\right) \text { for } x \geq 0, v \in \mathbb{R}
$$

given by [48] for $v=0$ and $x=0$ and [12] for general $x$ and $v$. That the higher-dimensional distributions of the process $\left(L_{1, v}^{\mathrm{br}}, v \in \mathbb{R}\right)$ are not so simple is clear already from the complexity of Proskurin's formula [66, 1, 72, 73] for the density of $L_{1, v}^{|\mathrm{br}|}=L_{1, v}^{\mathrm{br}}+L_{1,-v}^{\mathrm{br}}$ for $v>0$.

Let $-I$ denote the infimum of the standard bridge $B^{\text {br, } 1}$, so

$$
-I:=\inf _{0 \leq u \leq 1} B_{u}^{\mathrm{br}, 1}=\inf \left\{r: L_{1, r}^{\mathrm{br}}>0\right\} \quad \text { a.s. }
$$

As observed in [6],

$$
\left(L_{1, v-I}^{\mathrm{br}}, v \geq 0\right)=\left(L_{1, v}\left(B^{\mathrm{ex}, 1}\right), v \geq 0\right),
$$

where $B^{\mathrm{ex}, 1}$ is the standard Brownian excursion derived from $B^{\mathrm{br}, 1}$ by Vervaat's [75] transformation. Combined with Corollary 5, this yields the following description of the process of bridge local times.

Corollary 17.

$$
\left(L_{1, r}^{\mathrm{br}}, r \in \mathbb{R} ; I\right)=_{d}\left(X_{0,1,(J+r) \vee 0}, r \in \mathbb{R} ; J\right),
$$

where on the right side $P\left(\left(J \in d u \mid X_{0,1, v}, v>0\right)=X_{0,1, u} d u, u>0\right.$.

Proof. In view of (41) it suffices to show $P\left(I \in d u \mid B^{\mathrm{ex}, 1}\right)=$ $L_{1, u}\left(B^{\mathrm{ex}, 1}\right)(d u)$, and this follows easily from Biane's observation that the time of the minimum of $B^{\mathrm{br}, 1}$ is independent of $B^{\mathrm{ex}, 1}$ with uniform distribution on $[0,1]$.

See [14] for related results. As shown by Lévy, the random variable

$$
A_{1}^{\mathrm{br}}:=\int_{0}^{1} 1\left(B_{u}^{\mathrm{br}, 1} \geq 0\right) d u=\int_{0}^{\infty} L_{1, v}^{\mathrm{br}} d v
$$


has a uniform distribution on [0,1]. The joint distribution of $\left(L_{1,0}^{\mathrm{br}}, A_{1}^{\mathrm{br}}\right)$, while not as simple as its marginals, is in principle determined by transforms which can be read from Ray's description up to time $T_{\theta}$, or from the Feynman-Kac formula [26]. By consideration of the kind of transformation between reflecting and unreflecting bridges described in [5], Lemma 5.2, Theorem 4 yields also the following corollary.

Corollary 18. Conditionally given $\left(L_{1,0}^{\mathrm{br}}, A_{1}^{\mathrm{br}}\right)=(l, a)$, the processes $\left(L_{1, v}^{\mathrm{br}}\right.$, $v \geq 0)$ and $\left(L_{1,-v}^{\mathrm{br}}, v \geq 0\right)$ are independent copies of $\left(X_{l, a, v}, v \geq 0\right)$ and $\left(X_{l, 1-a, v}, v \geq 0\right)$, respectively.

7. Concluding remarks. Perkins [56] showed that for each fixed $t>0$ the process of local times of $B$ at levels $v$ up to time $t$ is a semimartingale as $v$ ranges over all real values, and he gave the semimartingale decomposition of this process. Jeulin [27] gave a version of Perkins results that allows conditioning on $B_{t}$. See also [74] and [47] for descriptions of Brownian local time processes at a fixed time which are closely related to the results of this paper. Presumably, a similar description of the process $\left(L_{t, v}(|B|), v \geq 0\right)$ could be obtained, and then Theorem 4 should appear after conditioning on $B_{t}=0$.

As remarked in the discussion below Theorem 7, the left side of formula (12) has no meaning for $l=0$ and $t>0$, even though the process $\left(X_{0, t, v}\right.$, $v \geq 0$ ) is a well-defined process identical in law to the process of local times of $B^{\mathrm{ex}, t}$, a Brownian excursion of length $t$. However, for $l>0$, (12) amounts to the following identity of probability measures on $C[0, \infty)$ :

$$
Q_{l}^{(0)}=\int_{0}^{\infty} Q_{l, t} q_{l}^{(0)}(d t)
$$

where $Q_{l}^{(0)}$ is the law of the $B E S Q_{l}^{(0)}$ process starting at $l>0$, where $Q_{l, t}$ is the law of $\left(X_{l, t, v}, v \geq 0\right)$ for $l \geq 0$ and $t>0$ and $q_{l}^{(0)}$ denotes the distribution of $\int_{0}^{\infty} X_{v} d v$ for $X$ with distribution $Q_{l}^{(0)}$, that is, for $t>0$,

$$
q_{l}^{(0)}(d t)=P\left(\tau_{l} \in d t\right)=\frac{l}{\sqrt{2 \pi}} t^{-3 / 2} \exp \left(-\frac{l^{2}}{2 t}\right) d t,
$$

where the first equality is read from the Ray-Knight theorem (11), and the second is Lévy's formula for the density of the stable(1/2) variable $\tau_{l}$. If this form (43) of formula (12) is divided by $l$, and the limit taken as $l \downarrow 0$, the result is the following corollary, where according to (5), the law $Q_{0, t}$ of $\left(X_{0, t, v}, v \geq 0\right)$ may be interpreted as the law of local times up to time $t$ of a Brownian excursion of length $t$, as in $[63,64]$.

CoRollary 19 (Pitman-Yor [63, 64]). The formula

$$
M:=\int_{0}^{\infty} Q_{0, t} \frac{t^{-3 / 2} d t}{\sqrt{2 \pi}}
$$

defines a $\sigma$-finite measure on $C[0, \infty)$ under which the coordinate process is Markovian with the BESQ(0) semigroup, with almost every path starting at 0. 
As shown in [63], (4.2), this $\sigma$-finite law $M$ is the distribution of the ultimate local time process $\left(L_{\infty, v}(\varepsilon), v \geq 0\right)$ for $\varepsilon$ an element of $C[0, \infty)$ subject to Itô's $\sigma$-finite law of Brownian excursions. See [46, 60, 63, 64] for various developments and applications of this result to the Lévy-Itô representation of squared Bessel and related processes.

Le Gall and Yor [46] educed from the Lévy-Itô representation of squared Bessel processes [63] that $B E S Q_{0}^{(\delta)}$ for $\delta \geq 0$, can be constructed as the process of ultimate local times $\left(L_{\infty, v}\left(Y^{(\delta)}\right), v \geq 0\right)$ of $Y^{(\delta)}$ constructed from a reflecting Brownian motion $|B|$ as $Y_{t}^{(\delta)}:=|B|_{t}+L_{t, 0}(|B|) / \delta$ for $t \geq 0$. Carmona, Petit and Yor [13] found a similar construction of $B E S Q_{x}^{(\delta)}$ for $x>0$, $\delta<0$. See also [57, 58]. Norris, Rogers and Williams [53] Theorem 2, showed that the distribution of a local time process derived from another kind of perturbed Brownian motion, with a drift depending on its local time process, can be characterized by a variation of the Bessel square SDE like (2), but with a different form of path-dependent drift coefficient $\delta_{v}(X)$. See also [79] and papers cited there for various other Ray-Knight type descriptions of Brownian local time processes and further references on this topic.

Acknowledgments. Thanks to David Aldous and Marc Yor for many stimulating discussion related to the subject of this paper. Thanks also to Phil Spector for programming Figure 1.

\section{REFERENCES}

[1] Aldous, D. and Pitman, J. (1994). Brownian bridge asymptotics for random mappings. Random Structures and Algorithms 5 487-512.

[2] Aldous, D. J. (1991). The continuum random tree I. Ann. Probab. 19 1-28.

[3] Aldous, D. J. (1991). The continuum random tree II: an overview. In Stochastic Analysis (M. T. Barlow and N. H. Bingham, eds.) 23-70. Cambridge Univ. Press.

[4] Aldous, D. J. (1993). The continuum random tree III. Ann. Probab. 21 248-289.

[5] Bertoin, J. and Pitman, J. (1994). Path transformations connecting Brownian bridge, excursion and meander. Bull. Sci. Math. 118 147-166.

[6] Biane, P. (1986). Relations entre pont et excursion du mouvement brownien réel. Ann. Inst. H. Poincaré 22 1-7.

[7] Biane, P. and YoR, M. (1987). Valeurs principales associées aux temps locaux browniens. Bull. Sci. Math. 111 23-101.

[8] Biane, P. and Yor, M. (1988). Sur la loi des temps locaux browniens pris en un temps exponentiel. Séminaire de Probabilités XXII. Lecture Notes in Math. 1321 454-466. Springer, Berlin.

[9] Blumenthal, R. M. (1983). Weak convergence to Brownian excursion. Ann. Probab. 11 $798-800$.

[10] Borodin, A. N. (1986). On the character of convergence to Brownian local time I. Probab. Theory Related Fields 72 231-250.

[11] Borodin, A. N. (1986). On the character of convergence to Brownian local time II. Probab. Theory Related Fields 72 251-277.

[12] Borodin, A. N. (1989). Brownian local time. Uspekhi Mat. Nauk. 44 7-48.

[13] Carmona, P., Petit, F. and Yor, M. (1994). Some extensions of the arc sine law as partial consequences of the scaling property of Brownian motion. Probab. Theory Related Fields 100 1-29.

[14] Chaumont, L. (1997). An extension of Vervaat's transformation and its consequences. Prépublication 402, Laboratoire de Probabilités, Univ. Paris VI. 
[15] Chung, K. L. (1996). Excursions in Brownian motion. Arkiv für Matematik 14 155-177.

[16] Drmota, M. and Gittenberger, B. (1997). On the profile of random trees. Random Structures Algorithms 10 421-451.

[17] Drmota, M. and Gittenberger, B. (1997). On the strata of random mappings - a combinatorial approach. Preprint. Stochastic Process. Appl. To appear.

[18] Dwass, M. (1969). The total progeny in a branching process. J. Appl. Probab. 6 682-686.

[19] FELleR, W. (1951). The asymptotic distribution of the range of sums of independent random variables. Ann. Math. Statist. 22 427-432.

[20] Feller, W. (1951). Diffusion processes in genetics. Proc. Second Berkeley Symp. Math. Statist. Probab. 227-246. Univ. California Press, Berkeley.

[21] HaRris, T. E. (1952). First passage and recurrence distributions. Trans. Amer. Math. Soc. 73 471-486.

[22] Imноғ, J. P. (1984). Density factorization for Brownian motion, meander and the three-dimensional Bessel process, and applications. J. Appl. Probab. 21 500-510.

[23] Imнof, J. P. and Kümmerling, P. (1986). Operational derivation of some Brownian motion results. Internat. Statist. Rev. 54 327-341.

[24] Iтô, K. (1971). Poisson point processes attached to Markov processes. Proc. Sixth Berkeley Symp. Math. Statist. Probab. 3 225-240. Univ. California Press, Berkeley.

[25] Iтô, K. and McKean, H. P. (1965). Diffusion Processes and Their Sample Paths. Springer, New York.

[26] Jeanblanc, M., Pitman, J. and Yor, M. (1997). The Feynman-Kac formula and decomposition of Brownian paths. Comput. Appl. Math. 16 27-52.

[27] Jeulin, T. (1985). Temps local et théorie du grossissement: application de la théorie du grossissement à l'étude des temps locaux browniens. Grossissements de filtrations: exemples et applications. Lecture Notes in Math. 1118 197-304. Springer, Berlin.

[28] KAWAZU, K. and WatanaBe, S. (1971). Branching processes with immigration and related limit theorems. Theory Probab. Appl. 16 36-54.

[29] Kennedy, D. P. (1976). The distribution of the maximum Brownian excursion. J. Appl. Probab. 13 371-376.

[30] Kersting, G. (1996). On the profile of a conditioned Galton-Watson process. Unpublished manuscript.

[31] Kersting, G. (1998). On the height profile of a conditioned Galton-Watson tree. Unpublished manuscript.

[32] Kiefer, J. (1959). $K$-sample analogues of the Kolmogorov-Smirnov and Cramér-von Mises tests.

[33] Knight, F. (1997). Approximation of stopped brownian local time by diadic upcrossing chains. Stochastic Process. Appl. 66 253-270.

[34] Knight, F. B. (1963). Random walks and a sojourn density process of Brownian motion. Trans. Amer. Math. Soc. 197 36-56.

[35] Knight, F. B. (1998). The moments of the area under reflected Brownian bridge conditional on its local time at zero. Preprint.

[36] Kolchin, V. F.. Random Mappings. Optimization Software, New York. (Trans. of Russian original.)

[37] Kurtz, T. G. and Protter, P. (1991). Weak limit theorems for stochastic integrals and stochastic differential equations. Ann. Probab. 19 1035-1070.

[38] Kushner, H. J. (1974). On the weak convergence of interpolated Markov chains to a diffusion. Ann. Probab. 2 40-50.

[39] Lamperti, J. (1967). Limiting distributions for branching processes. Proc. Fifth Berkeley Symp. Math. Statist. Probab. 2 225-241. Univ. California Press, Berkeley.

[40] Lamperti, J. (1967). The limit of a sequence of branching processes. Z. Wahrsch. Verw. Gebiete 7 271-288.

[41] Le Gall, J. F. (1986). Une approche élémentaire des théorèmes de décomposition de Williams. Séminaire de Probabilités XX. Lecture Notes in Math. 1204 447-464. Springer, Berlin. 
[42] Le GaLL, J. F. (1989). Marches aléatoires, mouvement Brownien et processus de branchement. Séminaire de Probabilités XXIII. Lecture Notes in Math. 1372 258-274. Springer, Berlin.

[43] Le GaLl, J. F. (1991). Brownian excursions, trees and measure-valued branching processes. Ann. Probab. 19 1399-1439.

[44] Le Gall, J. F. (1993). The uniform random tree in a Brownian excursion. Probab. Theory Related Fields 96 369-383.

[45] LE GALL, J. F. and LE JAN, Y. (1998). Branching processes in Lévy processes: the exploration process. Ann. Probab. 26 213-252.

[46] Le Gall, J. F. and Yor, J. (1986). Excursion browniennes et carrés de processus de Bessel. C. R. Acad. Sci. Paris Sér. I 303 73-76.

[47] Leuridan, C. (1998). Le théorème de Ray-Knight à temps fixe. Séminaire de Probabilités XXXII. Lecture Notes in Math. 1686 376-406. Springer, Berlin.

[48] LÉvy, P. (1939). Sur certains processus stochastiques homogènes. Compositio Math. 7 283-339.

[49] Lindval, T. (1972). Convergence of critical Galton-Watson branching processes. J. Appl. Probab. 9 445-450.

[50] McGiLl, P. (1986). Integral representation of martingales in the Brownian excursion filtration. Séminaire de Probabilités XX. Lecture Notes in Math. 1204 465-502. Springer, Berlin.

[51] Neveu, J. and Pitman, J. (1989). The branching process in a Brownian excursion. Séminaire de Probabilités XXIII. Lecture Notes in Math. 1372 248-257. Springer, Berlin.

[52] Neveu, J. and Pitman, J. (1989). Renewal property of the extrema and tree property of a one-dimensional Brownian motion. Séminaire de Probabilités XXIII. Lecture Notes in Math. 1372 239-247. Springer, Berlin.

[53] Norris, J. R., Rogers, L. C. G. and Williams, D. (1987). Self-avoiding random walk: a Brownian motion model with local time drift. Probab. Theory Related Fields $\mathbf{7 4}$ 271-287.

[54] Pavlov, Yu. L. (1988). Distributions of the number of vertices in strata of a random forest. Theory Probab. Appl. 33 96-104.

[55] Pavlov, YU. L. (1994). Limit distributions of the height of a random forest of plane rooted trees. Discrete Math. Appl. 4 73-88.

[56] Perkins, E. (1982). Local time is a semimartingale. Z. Wahrsch. Verw. Begiete 60 79-117.

[57] Perman, M. (1996). An excursion approach to Ray-Knight theorems for perturbed Brownian motion. Stochastic Process. Appl. 63 67-74.

[58] Perman, M. and Werner, W. (1998). Perturbed Brownian motions. Probab. Theory Related Fields 108 357-383.

[59] Pitman, J. (1975). One-dimensional Brownian motion and the three-dimensional Bessel process. Adv. in Appl. Probab. 7 511-526.

[60] Pitman, J. (1996). Cyclically stationary Brownian local time processes. Probab. Theory Related Fields 106 299-329.

[61] Pitman, J. (1997). Abel-Cayley-Hurwitz multinomial expansion associated with random mappings, forests and subsets. Technical Report 498, Dept. Statistics, Univ. California Berkeley. Available via http: // www. stat. berkeley. edu/ users / pitman.

[62] Pitman, J. (1998). Enumerations of trees and forests related to branching processes and random walks. In Microsurveys in Discrete Probability (D. Aldous and J. Propp, eds.) 163-180. Amer. Math. Soc., Providence, RI.

[63] Pitman, J. and Yor, M. (1982). A decomposition of Bessel bridges. Z. Wahrsch. Verw. Gebiete 49 425-457.

[64] Pitman, J. and Yor, M. (1996). Decomposition at the maximum for excursions and bridges of one-dimensional diffusions. In Itô's Stochastic Calculus and Probability Theory 293-310. Springer, New York.

[65] Pitman, J. and Yor, M. (1997). The two-parameter Poisson-Dirichlet distribution derived from a stable subordinator. Ann. Probab. 25 855-900. 
[66] Proskurin, G. V. (1973). On the distribution of the number of vertices in strata of a random mapping. Theory Probab. Appl. 18 803-808.

[67] RAY, D. B. (1963). Sojourn times of a diffusion process. Ill. J. Math. 7 615-630.

[68] Revuz, D. and Yor, M. (1994). Continuous Martingales and Brownian motion, 2nd ed. Springer, Berlin.

[69] Rogers, L. C. G. (1987). Continuity of martingales in the Brownian excursion filtration. Probab. Theory Related Fields 76 291-298.

[70] Rogers, L. C. G. and Walsh, J. B. (1991). The intrinsic local time sheet of Brownian motion. Probab. Theory Related Fields 88 363-379.

[71] Shiga, T. and WatanaBe, S. (1973). Bessel diffusions as a one-parameter family of diffusion processes. Z. Wahrsch. Verw. Gebiete 27 37-46.

[72] TAKÁCS, L. (1995). Brownian local times. J. Appl. Math. Stochastic Anal. 3 209-232.

[73] Takács, L. (1995). On the local time of the Brownian motion. Ann. Appl. Probab. 5 $741-756$.

[74] VAn Der Hofstad, R., Den Hollander, F. and König, W. (1997). Central limit theorem for the Edwards model. Ann. Probab. 25 573-597.

[75] VervaAt, W. (1979). A relation between Brownian bridge and Brownian excursion. Ann. Probab. 7 143-149.

[76] Williams, D. (1969). Markov properties of Brownian local time. Bull. Amer. Math. Soc. 75 1035-1036.

[77] Williams, D. (1970). Decomposing the Brownian path. Bull. Amer. Math. Soc. 76 871-873.

[78] Williams, D. (1974). Path decomposition and continuity of local time for one-dimensional diffusions I. Proc. London Math. Soc. (3) 28 738-768.

[79] Yor, M. (1992). Some Aspects of Brownian Motion I: Some Special Functionals. Birkhaüser, Boston.

DEPARTMENT OF STATISTICS

367 Evans HaLl \#3860

UNIVERSITY OF CALIFORNIA

BERKELEY, CALIFORNia 94720-3860

E-MAIL: pitman@stat.berkeley.edu 